\title{
Preparing the Workforce for Behavioral Health and Primary Care Integration
}

\author{
Jennifer Hall, MPH, Deborah J. Cohen, PhD, Melinda Davis, PhD, Rose Gunn, MA, \\ Alexander Blount, EdD, David A. Pollack, MD, William L. Miller, MD, MA, \\ Corey Smith, PsyD, Nancy Valentine, RN, PhD, and Benjamin F. Miller, PsyD
}

Purpose: To identify how organizations prepare clinicians to work together to integrate behavioral health and primary care.

Methods: Observational cross-case comparison study of 19 U.S. practices, 11 participating in Advancing Care Together, and 8 from the Integration Workforce Study. Practices varied in size, ownership, geographic location, and experience delivering integrated care. Multidisciplinary teams collected data (field notes from direct practice observations, semistructured interviews, and online diaries as reported by practice leaders) and then analyzed the data using a grounded theory approach.

Results: Organizations had difficulty finding clinicians possessing the skills and experience necessary for working in an integrated practice. Practices newer to integration underestimated the time and resources needed to train and organizationally socialize (onboard) new clinicians. Through trial and error, practices learned that clinicians needed relevant training to work effectively as integrated care teams. Training efforts exclusively targeting behavioral health clinicians (BHCs) and new employees were incomplete if primary care clinicians (PCCs) and others in the practice also lacked experience working with BHCs and delivering integrated care. Organizations' methods for addressing employees' need for additional preparation included hiring a consultant to provide training, sending employees to external training programs, hosting residency or practicum training programs, or creating their own internal training program. Onboarding new employees through the development of training manuals; extensive shadowing processes; and protecting time for ongoing education, mentoring, and support opportunities for new and established clinicians and staff were featured in these internal training programs.

Conclusion: Insufficient training capacity and practical experience opportunities continue to be major barriers to supplying the workforce needed for effective behavioral health and primary care integration. Until the training capacity grows to meet the demand, practices must put forth considerable effort and resources to train their own employees. (J Am Board Fam Med 2015;28:S41-S51.)

Keywords: Behavioral Medicine; Delivery of Health Care, Integrated; Primary Health Care; Qualitative Research

Behavioral health and primary care integration refers to primary care clinicians (PCCs) and behavioral health clinicians (BHCs) and staff working

This article was externally peer reviewed.

Submitted 9 February 2015; revised 27 February 2015; accepted 11 March 2015.

From the Department of Family Medicine (JH, DJC, $\mathrm{MD}, \mathrm{RG}$ ), and Department of Medical Informatics and Clinical Epidemiology (DJC.), Oregon Health \& Science University, Portland; Oregon Rural Practice-Based Research Network (MD), Portland; Department of Family Medicine and Community Health (AB), University of Massachusetts, Worcester; Department of Psychiatry (DAP), Oregon Health \& Science University, Portland; Department of Family Medicine (WLM), Lehigh Valley Health Network, Allentown, PA; MidValley Family Practice (CS), Basalt, CO; Department of Health Systems Science (NV), together with patients to address patients' primary care and behavioral health needs. ${ }^{1}$ As integrated care becomes a more widely adopted clinical ap-

Institute for Healthcare Innovation, Chicago, IL; and Department of Family Medicine (BFM), University of Colorado, Aurora.

Funding: This research was supported by grants from the Colorado Health Foundation (CHF-3848), Agency for Healthcare Research and Quality (8846.01-S01), Tides Foundation/California Mental Health Services Authority Integrated Behavioral Health Project (AWD-131237), and the Maine Health Access Foundation (2012FI-0009).

Conflict of interest: none declared.

Corresponding author: Jennifer Hall, MPH, Department of Family Medicine, Oregon Health \& Science University, 3181 SW Sam Jackson Park Road, FM, Portland, OR 97233 (E-mail: haljenni@ohsu.edu). 
proach, the need to prepare health care professionals with skills and competencies to work together is growing. ${ }^{2-4}$ Presently, most PCCs and BHCs receive professional training in discipline-specific silos and in relative isolation from 1 another., ${ }^{3,5,6}$ Although this is beginning to change, ${ }^{7,8}$ the dominant paradigm has created BHCs and PCCs with little or no training in working collaboratively to deliver shared, patient-centered care. ${ }^{3,9-11}$

The workforce competencies, skills, behaviors, and attitudes health professionals need to work in integrated settings has been articulated in previous studies and reports. ${ }^{7,9,12-18}$ This article highlights the recruitment and training challenges we observed and heard about from leaders, clinicians, and staff developing integrated care approaches in their own practices. We also describe the strategies, structures, and work processes practices adopted to address health professionals' inexperience and prepare practice teams to deliver integrated behavioral health and primary care.

\section{Methods \\ Sample}

The sample was purposefully chosen and included 19 U.S. practices currently integrating behavioral health and primary care. Eleven practices were participants in the Advancing Care Together (ACT) program, and 8 practices, selected as exemplars of integration by an advisory committee, were participants in the Integration Workforce Study (IWS). For more information on these samples, please see Cohen et $\mathrm{al}^{19}{ }^{19}$ in this issue. This research was supported by grants from the Colorado Health Foundation, Agency for Health Care Research and Quality, California Mental Health Services Authority Integrated Behavioral Health Project, and Maine Health Access Foundation.

\section{Data Collection}

We studied a group of 11 ACT practices over a 3-year study period between September 2011 and September 2014 and 8 IWS practices at a single point in time between December 2012 and October 2013. The same team collected data for both studies and used similar data collection tools and strategies. Data collection included a 2-to-5-day site visit in which multiple trained field researchers observed practice operations, including meetings and primary care and behavioral health care deliv- ery. The observation visits provided an opportunity to learn how practices prepared clinicians to work in integrated settings. In addition, field researchers conducted semistructured interviews with 8 to 12 practice members at each practice. During these interviews respondents shared their experiences and information about training and organizational socialization (onboarding) to prepare staff to work in the practice. For ACT, we also collected observational data over time as practices implemented locally developed integration strategies using an online diary or journaling tool. ${ }^{20}$

\section{Data Management and Analysis}

Interviews were professionally transcribed, and interview transcripts and field notes were deidentified and entered into Atlas.ti (Version 7.0, Atlas.ti Scientific Software Development, $\mathrm{GmbH}$ ) for data management and analysis. A multidisciplinary team analyzed data using an immersion-crystallization approach. ${ }^{21}$ Together, team members read field notes and interview transcripts and identified text relevant to training, recruitment, and orientation. The team reviewed the data together and built a complete list of codes before dividing the remaining field notes and transcripts. Team members independently read and coded the data, meeting regularly as a group to discuss emerging findings. The challenges of hiring staff experienced in integrated care emerged during this phase. During the second round of analysis we examined the organizational structures and processes practices developed to address these training challenges and prepare clinicians to work in integrated care. The Oregon Health \& Science University, the University of Colorado, and the University of Texas institutional review boards approved the study protocols.

\section{Results}

Participating practices varied in size, ownership, geographic location, and experience delivering integrated care. For more details on practice characteristics, see Cohen et al, ${ }^{19}$ in this issue. Across the 19 sites, we directly observed and heard from practice members about the difficulty of preparing clinicians and staff to work in integrated care settings. We describe these recruitment and training challenges and identify how organizations address professional development needs to promote effective delivery of integrated care: developing practicum 
Table 1. Observed Differences between Traditional Mental Health and Integrated Primary Care

\begin{tabular}{|c|c|}
\hline Traditional Mental Health & Integrated Behavioral Health and Primary Care \\
\hline 50-min appointments & Brief, targeted interventions ( 5 to $30 \mathrm{~min}$ ) \\
\hline $\begin{array}{l}\text { Asynchronous communication with other healthcare } \\
\text { stakeholders (eg, fax a note, voice message) }\end{array}$ & $\begin{array}{l}\text { Immediate communication with other members of the team: directly or } \\
\text { within the shared EHR }\end{array}$ \\
\hline Interventions often focused on mental health & $\begin{array}{l}\text { Interventions focused on behavioral health: mental health, substance use, } \\
\text { life stressors, health behaviors, and adherence to medical regimens }\end{array}$ \\
\hline $\begin{array}{l}\text { Clinical involvement often long term, likely to take } \\
\text { a reflective approach }\end{array}$ & $\begin{array}{l}\text { Clinical involvement focused on the moment (eg, problem and/or } \\
\text { solution), likely to take a more active and teaching approach }\end{array}$ \\
\hline Patients discharged following completion of care & Patients retained in the EHR as long as they are receiving primary care \\
\hline $\begin{array}{l}\text { Documentation often in narrative form: focused on } \\
\text { telling the person's history and story }\end{array}$ & $\begin{array}{l}\text { Documentation often brief, focused on problem, intervention, and plan, } \\
\text { and located either in separate note or imbedded in physicians' notes }\end{array}$ \\
\hline $\begin{array}{l}\text { Must document development of thorough } \\
\text { knowledge of client }\end{array}$ & Knowledge of patient developed by PCP in previous relationship \\
\hline Assign clinical diagnosis to bill & $\begin{array}{l}\text { Diagnosis often resisted or delayed to try to help the patient without a } \\
\text { label }\end{array}$ \\
\hline $\begin{array}{l}\text { Individuals referred to as "clients," "consumers," or } \\
\text { other term designed to reduce stigma }\end{array}$ & Individuals referred to as "patients" or "consumers" \\
\hline
\end{tabular}

EHR, electronic health record.

training programs; implementing rigorous onboarding practices for new clinicians; and developing ongoing learning, education and support opportunities for clinicians and staff.

\section{Recruitment Challenges}

Few organizations were able to hire clinicians who already possessed sufficient skills and experience to work in teams delivering care for a shared patient population in an integrated practice. For BHCs, this meant being adaptable to the pace and workflow of primary care clinics, knowing how to work with a PCC team; understanding the health care needs of a primary care population; and knowing how to use brief assessments, counseling, and solution-focused approaches with patients. Table 1 highlights these and other differences between delivering integrated behavioral health and primary care and traditional therapy approaches.

For PCCs, practicing in an integrated setting meant working on a care team with BHCs, consistently identifying patients' behavioral health and emotional needs, knowing when and how to involve BHCs, and being open to incorporating another professional's expertise into patient care. For psychiatrists, working in integrated settings meant consulting with PCCs and BHCs, conducting rapid, minimal assessments with patients directly, or using information from PCCs and other BHCs to inform decision making and treatment advice. Table 2 summarizes additional skills that PCCs,
BHCs, and psychiatrists needed to deliver integrated care.

In our interviews with practice leaders and decision makers we heard that when recruiting new employees, most did not find candidates who had developed these competencies and skills either in training or through previous work experience. In our observations of practices going through the hiring process, with few exceptions, most were unable to hire clinicians experienced in integrated care delivery.

\section{Training Challenges for Practices New to Integration}

For organizations with little experience in integration, hiring relatively inexperienced health care professionals meant that there were few opportunities to model or train new employees or to orient them to integrated care. Practice leaders also underestimated the time and resources required to train new clinicians: they often did not know what knowledge, skills, or attitudes were essential for new employees. In addition, practice leaders often failed to recognize this as a problem until after unsuccessful implementation. For example, practices might hire BHCs whose prior training and work experiences had only been with traditional mental health concepts or settings. BHCs were unable to effectively handle frequent interruptions by other clinic staff, provide brief assessments or interventions, consult with the primary care team 
Table 2. Summary of Observed Skills Clinicians Need to Deliver Integrated Care ${ }^{12}$

\begin{tabular}{|c|c|c|}
\hline PCC & $\mathrm{BHC}$ & Consulting Psychiatrist \\
\hline \multirow[t]{2}{*}{$\begin{array}{l}\text { Open to sharing care with others: } \\
\text { recognizes that other professionals } \\
\text { bring specialized expertise }\end{array}$} & $\begin{array}{l}\text { May proactively review patients to identify } \\
\text { potential needs/opportunities for BHC } \\
\text { services to PCC }\end{array}$ & $\begin{array}{l}\text { Views role as adjunct to PCC and BHC, } \\
\text { not as the replacement }\end{array}$ \\
\hline & $\begin{array}{l}\text { Participates in briefing by listening and } \\
\text { asking for clarification when needed }\end{array}$ & \\
\hline $\begin{array}{l}\text { Reviews screenings, uses clinical } \\
\text { discretion, or considers suggestions } \\
\text { to identify patients needing BHC } \\
\text { services }\end{array}$ & $\begin{array}{l}\text { Introduces self as a member of the care } \\
\text { team: normalizes behavioral health care } \\
\text { delivery as part of "routine" practice }\end{array}$ & $\begin{array}{l}\text { Introduces self as a member of the care } \\
\text { team }\end{array}$ \\
\hline $\begin{array}{l}\text { Provides brief assessment of patient's } \\
\text { behavioral health needs and } \\
\text { establishes foundation for BHC } \\
\text { handoff: describing expertise, } \\
\text { importance of whole person care, } \\
\text { expectations ("selling" resource to } \\
\text { patient) }\end{array}$ & $\begin{array}{l}\text { Conducts rapid, targeted assessments of } \\
\text { patient needs; identifies "feasible" targets } \\
\text { for brief intervention }\end{array}$ & $\begin{array}{l}\text { Provides psychiatric consultation to the } \\
\text { primary care team as needed }\end{array}$ \\
\hline $\begin{array}{l}\text { Briefs BHC on perceived patient } \\
\text { condition/needs: assessment of } \\
\text { situation, depression intervention }\end{array}$ & $\begin{array}{l}\text { Sets agenda balancing patient/PCC } \\
\text { priorities, or negotiates focus of these } \\
\text { two stakeholders }\end{array}$ & $\begin{array}{l}\text { Assists PCC: diagnosis, treatment } \\
\text { planning, and recommendations }\end{array}$ \\
\hline $\begin{array}{l}\text { Available to debrief with } \mathrm{BHC} \text { during } \\
\text { encounter or post-encounter to } \\
\text { develop care plan }\end{array}$ & $\begin{array}{l}\text { Apply brief interventions using abbreviated } \\
\text { evidence-based treatment strategies: } \\
\text { solution-focused therapy, behavioral } \\
\text { activation, cognitive behavioral therapy, } \\
\text { motivational interview }\end{array}$ & $\begin{array}{l}\text { Willing to treat/consult on some } \\
\text { patients without seeing them }\end{array}$ \\
\hline \multirow[t]{7}{*}{$\begin{array}{l}\text { Reinforces care plan and/or } \mathrm{BHC} \\
\text { intervention during next encounter }\end{array}$} & $\begin{array}{l}\text { Clinical capacity to address full spectrum of } \\
\text { behavioral health needs: common mental } \\
\text { health conditions (depression, anxiety), } \\
\text { lifestyle behaviors (self-care, social } \\
\text { engagement, relaxation, sleep hygiene, } \\
\text { diet, exercise) }\end{array}$ & $\begin{array}{l}\text { Makes a treatment plan in a short } \\
\text { amount of time with limited } \\
\text { information }\end{array}$ \\
\hline & $\begin{array}{l}\text { May develop specialization areas: substance } \\
\text { use counseling, biofeedback }\end{array}$ & $\begin{array}{l}\text { Focuses on complex patients who cannot } \\
\text { be managed alone by PCP and BHC }\end{array}$ \\
\hline & $\begin{array}{l}\text { Links efforts to overall patient care: } \\
\text { reinforce care plan with PCC and } \\
\text { summarize goals/next steps with patient }\end{array}$ & \\
\hline & $\begin{array}{l}\text { Leads group sessions for patients: pain } \\
\text { groups, diabetes management }\end{array}$ & $\begin{array}{l}\text { Engages other professionals in patient } \\
\text { care plan: BHC, social worker, } \\
\text { pharmacist }\end{array}$ \\
\hline & $\begin{array}{l}\text { Determines care level needed: additional } \\
\text { followup with BHC, transition to } \\
\text { specialty mental health care }\end{array}$ & $\begin{array}{l}\text { Coaches PCCs to manage complex } \\
\text { patients; transitions patients back to } \\
\text { primary care }\end{array}$ \\
\hline & $\begin{array}{l}\text { Assists with specialty mental health/other } \\
\text { treatment resource transitions (case } \\
\text { management) }\end{array}$ & \\
\hline & $\begin{array}{l}\text { Concisely communicates information to the } \\
\text { primary care team: verbally, EHR notes }\end{array}$ & $\begin{array}{l}\text { Concisely communicates information to } \\
\text { the primary care team verbally, EHR } \\
\text { notes }\end{array}$ \\
\hline
\end{tabular}

BHC, behavioral health clinician; EHR, electronic health record; PCC, primary care clinician.

about patient needs and care provided, or concisely document visit notes in the electronic health record (EHR). Practice leaders eventually learned through trial and error, and in some cases from rapid turnover in the BHC position, that specialty mental health clinicians needed relevant training to work effectively with primary care teams. For example, 1 ACT practice, collaborating with a local community mental health center, placed a BHC in their practice. The practice reported the following:
As [BHC] writes notes and puts her ideas for treatment together, she has found it extremely difficult to leave the world of behavioral health and integrate patient and medical ideas. As much as I have tried to teach her about what patient centric means, I truly do not think she understands. After much training she still wants to revert to writing oldstyle behavioral health notes. There are 
many more examples. Next time around I wonder if I can train better. (Diaries, Practice 9)

Other practices had similar experiences, and some reported frequent turnover with their BHCs, which led to some practices refining their $\mathrm{BHC}$ training approaches.

In addition, the loss of multiple BHCs led to the development of reciprocal training for PCCs and staff to learn to work with BHCs. Practices underestimated the time and resources needed to recalibrate and retrain current clinicians and staff to work using an integrated care approach. Training efforts that exclusively targeted BHCs or new employees were incomplete if PCCs and others in the practice lacked experience working with BHCs and delivering integrated care. PCCs needed to learn to make appropriate referrals to BHCs, adjust their workflow to accommodate warm handoffs, and incorporate other clinicians' expertise into patient care.

\section{Practice Strategies for Preparing an Integrated Care Workforce}

We observed a variety of approaches to preparing clinical staff to deliver integrated care. These included relying on an internal expert to provide training, sending employees to external training programs, or developing internal postdoctoral or practicum training programs. Once organizations identified the need to prepare everyone in the practice-not just new employees-in delivering integrated care, organizations addressed this training need:

And primary care- they think so differently than $\mathrm{MH}$ [mental health] people! Even if they say they understand what we're doing, they do not! So I talked with [the practice manager] about doing some training with all the staff-not just clinicians but ALL the staff. I did some training on brief therapy and problem solving. These were small little videos I did. Then I just sat and talked with them and answered questions. Each training was an hour. I went to 3 team meetings every other week-about 3 times overall. Given that the BHC is starting we're going to look at what needs to be done and if she can help with some of the trainings. It was really helpful. Just sitting down and talking with them, answering questions. (Diaries, Practice 10)

When practices lacked the internal experience to train employees in integrated care, they could hire consultants to offer onsite training or send employees to external training programs. Some organizations we studied hosted such trainings with clinicians and organizational leaders, and regularly provided tours to demonstrate how they provide integrated care. However, sending employees to external training programs potentially limits the number of individuals who can attend and can be resource prohibitive in terms of training costs, travel expenses, and loss of revenue-producing work time. The following excerpt from a prefunding interview with an ACT practice describes the perception of the costs of formal training for clinicians in integrated care:

The certification took 7 to 8 full days across 4 months. If we elect to use this model it would be exceptionally difficult to cover patient care and maintain revenue with all physicians being trained at the same time. The training for all PCCs and BHCs would cost $\$ 80,000$, which is financially unrealistic for a private clinic. Currently, some of the previously trained physicians and clinicians in this model will be participating in an update webinar. These are necessary but also time consuming and must either be done on days off or during patient care hours. (Evaluability Assessment Notes, Practice 13)

Another way organizations tried to increase workforce expertise was to offer to be a postgraduate practicum site for medical (eg, primary care or psychiatry) residencies and behavioral health disciplines (eg, psychology or social work) graduates to meet supervised clinical experience requirements for licensure. Program trainees gained hands-on experience working in integrated care teams and received training and supervision from a multidisciplinary team of clinicians. For example, medical residents' clinical encounters were reviewed by 
BHCs, psychiatrists, and/or pharmacists. Behavioral health students gained consultation skills, confidence, and comfort working directly with PCCs, as well as therapy and intervention skills applicable in primary care settings by working with highly experienced BHCs:

One of the biggest things [I teach my behavioral health students] is to not be afraid to go and talk to another provider. Because I think that they are intimidated. I know that I was when I first started in this kind of a setting... You see the medical providers as being way up here. And then coming in as an intern, you know, I am going to walk into the doctors' room and say I need to speak to you? So you need to have a voice and you need to go in there if you have a patient and there's a medication issue, to knock and say, this is who I am. I am working with this patient, and feeling like that is part of your job. You have to be able to do that. (Interview, Practice 1)

Providing postgraduate or practicum training within the practice required experienced personnel to supervise and teach trainees. These programs benefited the organizations by providing affordable options for uninsured patients but also helped with recruitment as it created a pipeline of highly qualified future applicants prepared to work in integrated clinics. Organizations with postgraduate training programs reported hiring a number of their former trainees:

With psychologists, we have the American Psychological Association (APA)approved interns, and there are 4 of those. That has been a real rich tool for us to be able to recruit BHCs. Because when they come here, they get the training to do that, and we get to pick who we would like and make an effort to recruit them. Do not hold me to this, but I'd say over the years we probably recruited half of them into [the organization], maybe more than half. (Interview, Practice 15)
Table 3. Common Integrated Care Training Manual Components

\begin{tabular}{l}
\hline Component \\
\hline Organizational background \\
Organizational history \\
Organization mission and vision \\
Information on the integration model (current and ideal) \\
Description of roles and responsibilities \\
Information for all clinicians \\
Sample script and handouts for introducing the model and \\
BHCs to patients \\
Team meeting descriptions and types \\
Smart phrases for the EHR \\
Screener forms \\
Coding and billing procedures \\
Information scheduling, appointment types, and duration \\
Health system (internal) and community (external) \\
resources \\
Workflow descriptions \\
Recommended references (articles, books, and websites) \\
Information for BHCs \\
Behavioral health interventions and treatment modalities \\
Descriptions of the differences between integrated care \\
provided by a BHC and specialty mental health \\
Documentation requirements and examples \\
Medical vocabulary \\
\hline
\end{tabular}

EHR, electronic health record.

\section{Onboarding Processes for New Employees}

Onboarding refers to how organizations socialize employees to the practice's culture, models of care, and workflow. Onboarding helps new staff obtain the knowledge, skills, and behaviors needed to effectively deliver care. Practices with more integrated care experience have developed extensive onboarding processes for new employees.

A key element of this process is the use of training manuals. As detailed in Table 3, training manuals were important reference guides for new employees. Training manuals included information on workflows, protocols, policies, and procedures as well as information on the history, culture, and vision of the organization:

It has everything in it from regular employment information like asking for time off and benefit information to information on the integration model, info on the BHC's role, contrast between $\mathrm{BHC}$ and integrated care with specialty mental health, visit types, team 
meeting descriptions and types, standards of practice, interventions and treatment modalities, smart phrases in Epic. (Field Notes, Practice 4)

Practice members identified the process of manual creation as pivotal in their own development, reinforcing their commitment to integration and encouraging reflection and consensus building on vision and values, as well as clinic processes.

Experienced clinics also created rigorous shadowing processes to onboard new employees. Considered an investment by the organizations, shadowing included observing professionals in the role one was hired to do, as well as roles with which new employees would be expected to collaborate. This helped clinicians and staff understand patient resources, the greater continuum of care, and how other positions interfaced with their roles and workflows. BHCs shadowed the PCCs with whom they would later work on care teams. Shadowing began the process of developing rapport and trust between clinicians who would work together:

Once they hire someone the new hire will shadow in all the clinics. It can feel cumbersome, but this is the best way to help prepare BHCs. New BHCs shadow the case manager, MA [medical assistant], and PCC so they understand the different workflows. Then they are shadowed by [the lead BHC supervisor] or another supervising $\mathrm{BHC}$ to provide feedback and make sure they are operating under their model. BHCs also meet with all new staff to describe and explain their role to new staff and clinicians. (Field Notes, Practice 5)

When [BHCs are] onboarded, they have the opportunity to shadow inpatient services and shadow day treatment, so they can see what the continuum of care is for their patients. So if they have a patient who went inpatient, they know what the daily routine is like, who they are going to meet with and what kind of groups they are going to participate in. They understand what day treatment is and what they can expect from that service. So, as you are talking about referring your patient to those services, you can give them a nice picture of what it is going to be like and take away some of the fears. And also understand what you can expect of your colleagues when they are receiving those services. (Field Notes, Practice 4)

The duration of shadowing varied among the practices from a few days to a few weeks. This depended on the practices' routines for onboarding, the type of employee, and the new employee's level of experience. In some practices after shadowing was complete, a supervisor or mentor would observe newly hired BHCs and PCCs before they practiced independently. Following this observation phase, new PCCs and BHCs were often given a lighter schedule to develop new skills, adapt to the team's workflow, and learn to use the EHR:

They do a lot of shadowing in their own
clinic. New clinicians then see patients
independently and get 60 -minute ap-
pointments. This gives them time to
learn the EHR and work with the
BHCs and the nutritionists, get used to
the flow, and stay on schedule while
integrating other components of care.
They also troubleshoot if anyone is
struggling with any of these compo-
nents. (Field Notes, Practice 4)

Following the shadowing and observation phases, supervisors remained available to new clinicians for curbside consultations, second opinions, and assistance with challenging patients. Supervisees were encouraged to ask questions and regularly received feedback from their supervisors:

We also believe heavily in the value of on-the-fly supervision. We are always available to them, especially initially when they are new to us. There's times early on we're consulting on the fly after every patient they are seeing. And sometimes a couple of times before they let the patient go, because we need to know, what do I need to tell this primary care provider? What should my plan be? What do I need to do with this patient? So we're kind of modeling for 
them, we're teaching them didactically. We're teaching them in supervision, we're consulting with them during the day. (Interview, Practice 2)

\section{Continuing Education and Support}

Organizations provided ongoing development and learning opportunities to existing personnel through the use of multidisciplinary meetings in which clinicians and staff discussed operational issues such as clinic policies or workflow changes. This time was also used for presentations led by various members of the care team. For example, psychiatrists or pharmacists held topic-specific trainings or open question-and-answer sessions for PCCs and BHCs. Presentation topics were based on clinician interests and requests. A psychiatrist who talked about her availability and answered basic questions regarding psychiatric issues led the following meeting:

The psychiatrist tells the group she wants to make sure they know she does consults Wednesday with obstetrics. Thursday she is in the telemedicine room 'embedded' in the pod there for consults, and then other days she is always available for questions or consults if needed. She wants to open the floor to see if there are any basic questions people have about psychiatry issues. An internist in the primary care practice has his laptop in front of him-he's got some questions prepared. He wonders if there is the Patient Health Questionnaire-2 equivalent for disorders other than depression-thought disorder, bipolar disorder for instance. He is looking for some 'key questions' he can ask to try and screen for these types of things. (Field Notes, Practice 2)

Organizations also developed formal, ongoing mentoring opportunities for peers to continuously support and learn from each other. After the onboarding process, various methods of mentoring were available to both new employees and to existing personnel. Mentors were often organization leaders with both administrative and clinical roles who modeled behaviors for integrating care and, by doing so, were not only a source of information and learning but, through their actions and behaviors, reinforced the integration model in the practice:

\begin{abstract}
All our administrative folks, heads, who are licensed as clinicians still carry caseloads.... I think that sets a tone, a recognition of if you are going to do the work or if you are going to administer the work, you have got to know what doing the work feels like and stay active with it. (Interview, Practice 2)
\end{abstract}

Other practices established ongoing meetings that allowed PCCs or BHCs to collaboratively review complex cases. Some practices used this time to engage in continuing education opportunities such as literature reviews on clinical topics. For example, a group of PCCs and BHCs reviewed literature together on physical activity and patients with Alzheimer disease:

The meeting continues with someone presenting on a study about physical activity and Alzheimer's. The study found that patients who participated in moderate exercise were $38 \%$ less likely to develop Alzheimer's. Another person in the group then reported on other benefits of physical activity. She shared an article from Health and Fitness magazine that explained the health benefits of exercise and strength training-including being good for depression. A PCC asks how this was different from what we already know about physical activity. Here it focused more on the benefits of strength training instead of aerobic activity like most materials tend to address. She thought it would be helpful to share with patients because it suggests that the 30 minutes of activity do not have to be done consecutively. This seems especially important because for some of their patients, starting at 10 minutes at a time is much less overwhelming. (Field Notes, Practice 15)

Opportunities for intra-organizational continuing education were limited and especially challenging for smaller practices without the capacity to 
provide peer support or educational meetings. This problem was compounded when organization leaders and clinicians in smaller practices lacked experience in integration and were not connected to peers outside of the organization with whom they could consult.

\section{Discussion}

We observed that both integration exemplars and those just getting started had difficulty recruiting BHCs and PCCs with experience in integrated care. There was a significant gap between clinicians' competencies (derived from academic or professional training and prior work experience) and practices' integrated workforce needs. Organizations and practices experienced difficulty recruiting BHCs with sufficient skills, experience and capacity to help change practice patterns to work effectively in a new culture of primary care. In addition, practices underestimated the training needs for PCCs and staff who also needed to learn to work collaboratively with BHCs.

Training issues were particularly perplexing for practices new to integration, given that individuals responsible for implementing integrated care and hiring new clinicians also lacked the knowledge and experience to arrange or provide necessary training. Through trial and error, practice leaders learned the ineffectiveness of expecting individuals with prior training only in segregated approaches to care, to function effectively in an integrated care setting without additional preparation. Without collaborative training experiences, PCCs and BHCs struggled to appreciate their teammates' work, clinical perspectives, pace of care, and patient needs in integrated settings.

Unfortunately, few primary care or behavioral health training programs provide the training necessary for clinicians to work in integrated practices. ${ }^{3,11,22}$ Moreover, BHCs and PCCs are infrequently trained in collaboration or practicing in interprofessional settings. ${ }^{6,17}$ Although increasing numbers of programs are emerging offering integrated care training for students, ${ }^{6,11,23-26}$ medical residents, ${ }^{6,11,24,27,28}$ or postdoctoral trainees, ${ }^{3}$ insufficient training capacity and practical experience opportunities continue to be major barriers to supplying the workforce needed for effective behavioral health and primary care integration. As practices and payers increasingly recognize the benefits of integrated care, the need and demand for competent clinicians to work in these integrated settings will grow. Until the training capacity grows to meet the demand, practices will have little choice but to train their own employees by creating their own training programs, hiring consultants, or accessing national training programs for clinicians and staff.

While national training programs have been evaluated using a methodology that makes a good case for improvement in skills, self-made programs have not been validated in the same way. In addition, what is learned in postgraduate training and practicum sites depends on the strength and quality of the model at the practice, and even individuals who are trained in integration might have a wide range of skill, understanding, and perspectives. Thus, standardization of the skills and competencies needed to work in an integrated model of care is needed.

Our findings suggest that practices frequently underestimate the time and expenses associated with training and onboarding new clinicians and staff. More information on startup and ongoing expenditures are described in Wallace et $\mathrm{al}^{29}$ in this issue. Although most practices emphasized the value of proper training for new staff, these options all require expertise, time, and money-creating additional barriers for smaller practices adopting an integrated model, which are unlikely to have the staff capacity and expertise to create their own programs. Lack of accurate knowledge about training programs or biased attitudes (ie, cost and benefit of effective training and value of asynchronous or distance-learning, mediated training), may create the belief that such training opportunities are not viable options, resulting in ill-conceived internal efforts to train employees or in abandoning the idea of providing any training at all.

Our findings show that regardless of training in integration, practices still needed to onboard new employees to understand the practice's integration model, the roles and responsibilities of other care team and health system members, and practice workflows. New employees need time to develop trust and rapport with their clinician teams. Shadowing allows clinicians to accomplish these relationship orientation functions as well as to familiarize new staff with essential work processes, including EHRs and personnel policies, in ways that create less pressure than starting work with a full patient panel without transition time. 
In addition to onboarding, some organizations created ongoing learning processes and recognized that learning was not limited to newly hired employees. These organizations developed structures that provided continuing education opportunities through mentorship and multidisciplinary meetings with the additional benefit that professionals will probably trust and learn from one another. Ongoing learning is especially important in organizations implementing innovative and evolving care models of care.

There are some limitations to these studies including selection and observer bias; however, these biases may be mitigated by having a multi-disciplinary team collect and analyze the data, and many of the training issues we observed are likely to be present among other practices integrating behavioral health and primary care. A major overarching issue that health care reform efforts have raised is the capacity, competence, and sustainability of the workforce. ${ }^{30-32}$ Numerous researchers and scholars have articulated the competencies that health professionals need to work in integrated care settings. ${ }^{7,17}$ Our study contributes to this literature through direct observation of how organizations with varying amounts of prior experience integrating behavioral health and primary care address the limited capacity of health professionals who are competent and ready to work together in integrated care teams.

\section{Conclusion}

Clinicians lack appropriate training and experience needed for effective behavioral health and primary care integration. Academic and educational training programs have not met the demand for a workforce that is competent in integrated care. Therefore, organizations must put forth considerable energy to prepare and support clinicians to work in these settings. Their extensive efforts and use of resources highlight the increasing need for academic and educational programs to train and prepare clinicians to work collaboratively and deliver care in integrated practices.

The authors are thankful to the 19 practices that participated in these studies. We appreciate data collection assistance from Emma Gilchrist, MPH and Leah Baruch, MD as well as assistance from Larry Green, MD; Frank deGruy III, MD; Sheldon Levy, PsyD; Mr. David Cameron; Ms. Claire Diener; and Ms. LeNeva Spires in the preparation of this manuscript.

\section{References}

1. Peek CJ, National Integration Academy Council. Lexicon for behavioral health and primary care integration: Concepts and definitions developed by expert consensus, 2013.

2. Fisher L, Dickinson WP. Psychology and primary care: New collaborations for providing effective care for adults with chronic health conditions. Am Psychol 2014;69:355-63.

3. Blount FA, Miller BF. Addressing the workforce crisis in integrated primary care. J Clin Psychol Med Settings 2009;16:113-9.

4. Rozensky R. Implications of the Affordable Care Act for education and training in professional psychology. Train Educ Prof Psychol 2014;8:83-94.

5. Kathol RG, Butler M, McAlpine DD, Kane RL. Barriers to physical and mental condition integrated service delivery. Psychosom Med 2010;72: 511-8.

6. Cubic B, Mance J, Turgesen JN, Lamanna JD. Interprofessional education: Preparing psychologists for success in integrated primary care. J Clin Psychol Med Settings 2012;19:84-92.

7. McDaniel SH, Grus CL, Cubic BA, et al. Competencies for psychology practice in primary care. Am Psychol 2014;69:409-29.

8. Beacham AO, Kinman C, Harris JG, Masters KS. The patient-centered medical home: Unprecedented workforce growth potential for professional psychology. Prof Psychol Res Pr 2012;43:17-23.

9. O’Donohue WT, Cummings NA, Cummings JL. The unmet educational agenda in integrated care. J Clin Psychol Med Settings 2009;16:94-100.

10. Pomerantz AS, Corson JA, Detzer MJ. The challenge of integrated care for mental health: leaving the 50 minute hour and other sacred things. J Clin Psychol Med Settings 2009;16:40-6.

11. Bluestein D, Cubic BA. Psychologists and primary care physicians: A training model for creating collaborative relationships. J Clin Psychol Med Settings 2009;16:101-12.

12. Cohen DJ, Davis MM, Hall JD, Gilchrist EC, Miller BF. A guidebook of professional practices for behavioral health and primary care integration: Observations from exemplary sites. Rockville, MD: Agency for Healthcare Research and Quality, 2014.

13. Miller WL, Cohen-Katz J. Creating collaborative learning environments for transforming primary care practices now. Fam Syst Health 2010;28:334-47.

14. Hunter CL, Goodie JL. Operational and clinical components for integrated-collaborative behavioral healthcare in the patient-centered medical home. Fam Syst Health 2010;28:308-21.

15. Hoge MA, Morris JA, Laraia M, Pomerantz A, Farley T. Core competencies for integrated behavioral health and primary care. Washington, DC: SAMHSA-HRSA Center for Integrated Health Solutions, 2014. 
16. Brown JD, Wissow LS. Rethinking the mental health treatment skills of primary care staff: A framework for training and research. Adm Policy Ment Health 2012;39:489-502.

17. Interprofessional Education Collaborative Expert Panel. Core competencies for interprofessional collaborative practice: Report of an expert panel. Washington, DC: Interprofessional Education Collaborative, 2011.

18. O'Donohue WT. Integrated care: whom to hire and how to train. In: James LC, O'Donohue WT, eds. The primary care toolkit. New York: Springer, 2009.

19. Cohen D, Balasubramanian B, Davis M, et al. Understanding care integration from the ground up: five organizing constructs that shape integrated practices. J Am Board Fam Med 2015;28:S7-S20.

20. Cohen DJ, Leviton LC, Isaacson N, Tallia AF, Crabtree BF. Online diaries for qualitative evaluation: Gaining real-time insights. Am J Eval 2006;27: 163-84.

21. Borkan J. Immersion/crystallization. In: Crabtree BF, Miller WL, eds. Doing Qualitative Research, 2nd ed. Thousand Oaks, CA: Sage Publications, 1999;179-94.

22. Blount A, DeGirolamo S, Mariani K. Training the collaborative care practitioners of the future. Fam Syst Health 2006;24:111-9.

23. Funderburk JS, Fielder RL. A primary mental health care model for advanced practicum training in a university health clinic. Train Educ Prof Psychol 2013;7:112-22.

24. Hoover M, Andazola J. Integrated behavioral care training in family practice residency: Opportunities and challenges. J Clin Psychol Med Settings 2012; 19:446-50.

25. Newton TL, Woodruff-Borden J, Stetson BA. Integrating mind and body: Graduate psychology education in primary behavioral health care. J Clin Psychol Med Settings 2006;13:3-11.

26. Twilling LL, Sockell ME, Sommers LS. Collaborative practice in primary care: Integrated training for psychologists and physicians. Prof Psychol Res Pr 2000;31:685-91.

27. Garfunkel LC, Pisani AR, leRoux P, Siegel DM. Educating residents in behavioral health care and collaboration: Comparison of conventional and integrated training models. Acad Med 2011;86:174-9.

28. Pisani AR, leRoux P, Siegel DM. Educating residents in behavioral health care and collaboration: Integrated clinical training of pediatric residents and psychology fellows. Acad Med 2011;86:166-73.

29. Wallace N, Cohen DJ, Gunn R, et al. Start-up and ongoing practice expenses of behavioral health and primary care integration interventions in the Advancing Care Together (ACT) program. J Am Board Fam Med 2015;28:S86-S97.

30. Miller BF, Petterson S, Burke BT, Phillips RL Jr, Green LA. Proximity of providers: Colocating behavioral health and primary care and the prospects for an integrated workforce. Am Psychol 2014;69: 443-51.

31. Petterson SM, Liaw WR, Phillips RL Jr, Rabin DL, Meyers DS, Bazemore AW. Projecting US primary care physician workforce needs: 2010-2025. Ann Fam Med 2012;10:503-9.

32. Robiner WN. The mental health professions: Workforce supply and demand, issues, and challenges. Clin Psychol Rev 2006;26:600-25. 